\section{JTI}

JOURNAL OF

TRAUMA AND INJURY

\title{
Non-Operative Management of Traumatic Gallbladder Bleeding with Cystic Artery Injury: A Case Report
}

\author{
Tae Hoon Kim, M.D.
}

Department of Emergency Medicine, Trauma Center, Wonkwang University Hospital, Iksan, Korea

Received: January 8, 2021

Revised: June 28, 2021

Accepted: July 5, 2021

\section{Correspondence to}

Tae Hoon Kim. M.D.

Department of Emergency Medicine, Trauma Center, Wonkwang University Hospital, 895 Muwang-ro, Iksan 54538, Korea

Tel: $+82-63-859-1650$

Fax: +82-63-859-1197

E-mail: doctor78em@gmail.com

ORCID: https://orcid.org/0000-00027683-7259
Gallbladder injuries are rare in cases of blunt abdominal trauma and are usually associated with damage to other internal organs. If the physician does not suspect gallbladder injury and check imaging studies carefully, it may be difficult to distinguish a gallbladder injury from gallbladder stone, hematoma, or bleeding. Therefore, in order not to miss the diagnosis, the clinical findings and correlation should be confirmed. In the present case, a 60-year-old male presented to a local trauma center complaining of pain in the upper right quadrant and chest wall following a motor vehicle collision. Abdominal computed tomography (CT) showed a hepatic laceration and hematoma in the parenchyma in segments 4, 5, and 6 and active bleeding in the lumen of the gallbladder. Traumatic gallbladder injuries generally require surgery, but in this case, non-operative management was possible with cautious follow-up consisting of abdominal CT and angiography with repeated physical examinations and hemodynamic monitoring in the intensive care unit.

Keywords: Gallbladder; Abdominal injury; Hemoperitoneum; Therapeutic embolization

\section{INTRODUCTION}

Traumatic gallbladder injuries are rare, and they are reported to account for approximately $2 \%$ to $11 \%$ of blunt abdominal injuries [1-4]. Their low incidence results from the gallbladder being protected by other adjacent organs, such as the liver, intestines, omentum, and ribs. Traumatic gallbladder injuries should be treated immediately, since the risk of serious complications or mortality increases if treatment is delayed [5-8]. Laparoscopy is usually the most valuable tool for the diagnosis and treatment of (http://creativecommons.org/licenses/by-nc/4.0/) which permits unrestricted noncommercial use, distribution, and reproduction in any medium, provided the original work is properly cited. 
penetrating or blunt trauma after inconclusive imaging [2]. In particular, traumatic gallbladder injuries are usually diagnosed on laparotomy when emergency surgery is necessary for other organ injuries [7-9]. However, advanced imaging modalities, such as high-speed computed tomography (CT) and ultrasonography, can be used to detect intraperitoneal fluid or hemorrhage and bile juice.

\section{CASE REPORT}

A 60-year-old male who had been involved in a motor vehicle collision presented to a local trauma center in a hemodynamically unstable condition with pain in the upper right quadrant area and chest wall. His mental state was slightly drowsy; he exhibited a Glasgow Coma Scale score of 13 (eye opening: 3, verbal response: 5 , and motor response: 5), and his vital signs were judged as indicating class II trauma (blood pressure, 100/70 mmHg; pulse rate, 106 beats/min; respiratory rate, 22
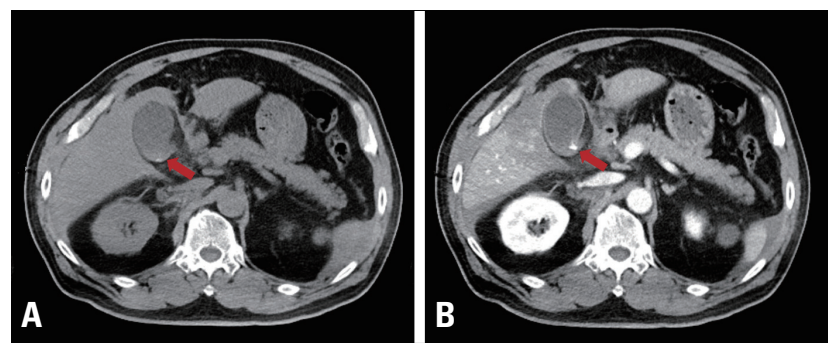

Fig. 1. (A) Hematoma and sludge in the gallbladder lumen on abdominal computed tomography (non-enhanced) (arrow). (B) Liver laceration with intraparenchymal hematoma and active bleeding in the gallbladder on abdominal computed tomography (enhanced) (arrow).

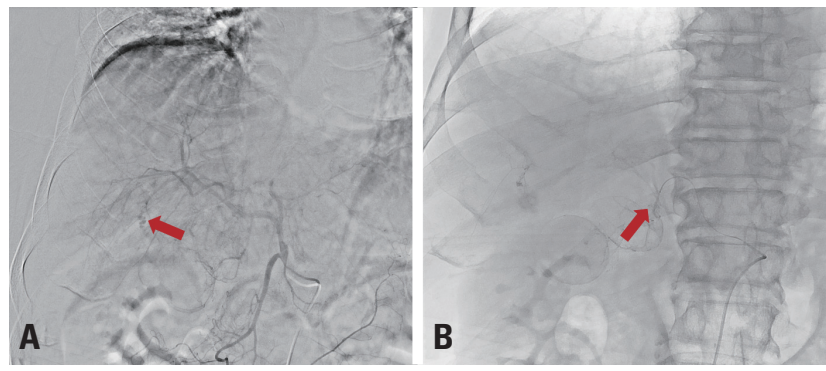

Fig. 2. (A) Contrast extravasation with pseudoaneurysm in the hepatic A5 branch and gallbladder (arrow). (B) Embolization after selection of the hepatic A5 culprit branch without cystic artery embolization (arrow). breaths/min; body temperature, $36.2^{\circ} \mathrm{C}$ ). The trauma team performed a focused assessment with sonography in trauma examination to identify abdominal fluid, and the findings revealed positive results in Morison's pouch and the perihepatic area. Contrast-enhanced abdominal CT suggested a liver laceration, with organ injury scale grade III and intraparenchymal subcapsular hematoma in segments 4, 5, and 6 and active bleeding in the gallbladder due to injury (Fig. 1). Since the patient's vital signs were unstable, as a part of non-operative treatment, hepatic angioembolization in the A5 culprit area was performed to establish hemorrhagic control (Fig. 2A). Focal contrast extravasation was observed on cystic arteriography, but it was decided to proceed with close observations without embolization (Fig. 2B). Forty-five hours (hospital day 3) after admission, repeated $\mathrm{CT}$ of the abdomen was
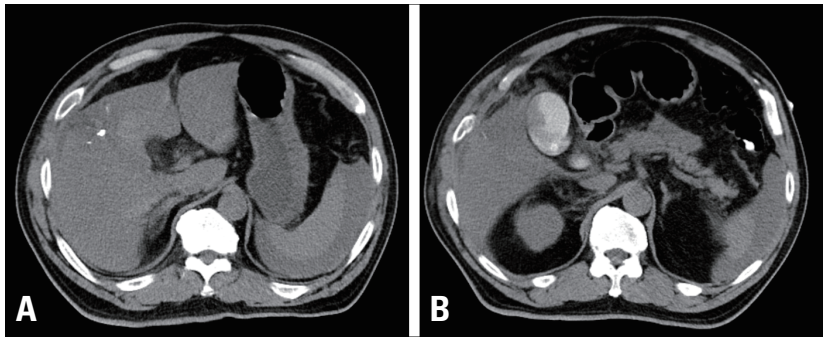

Fig. 3. (A) Increased amount of hemoperitoneal fluid in the perihepatic, perisplenic, and both paracolic areas on abdominal computed tomography (non-enhanced). (B) Heterogeneous contrast filling in the gallbladder, which indicates a multi-stage hematoma without active bleeding on abdominal computed tomography (enhanced).
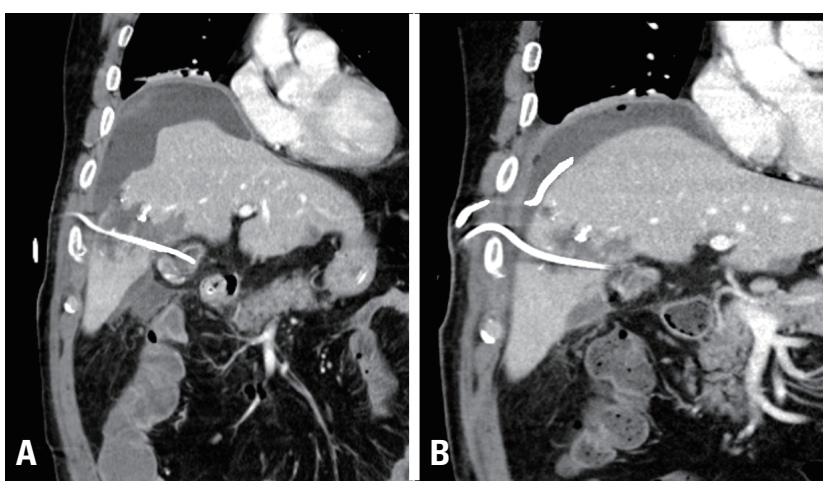

Fig. 4. (A) Percutaneous gallbladder drainage (PTGBD) insertion on the third day after angioembolization. (B) On hospitalization day 27, a decreased amount of perihepatic fluid and collapsed gallbladder without inflammation are observed after PTGBD and percutaneous drainage insertion. 
Table 1. Results of liver profile analysis

\begin{tabular}{|c|c|c|c|c|c|c|}
\hline & Initial & 24 hours & HD \#3 & HD \#5 & HD \#7 & HD \#14 \\
\hline AST (IU/L) & 468.0 & 955.0 & 881.0 & 406.0 & 154.0 & 31.0 \\
\hline ALT (IU/L) & 142.0 & 251.0 & 298.0 & 231.0 & 151.0 & 23.0 \\
\hline ALP $(I U / L)$ & 57.0 & 54.0 & 64.0 & 77.0 & 72.0 & 86.0 \\
\hline Bilirubin (mg/dL) & 0.5 & 0.7 & 1.3 & 2.3 & 1.7 & 0.5 \\
\hline Amylase (IU/L) & 86.0 & 48.0 & 31.0 & 26.0 & 29.0 & 66.0 \\
\hline Lipase (IU/L) & 84.0 & 27.0 & 15.0 & 14.0 & 24.0 & 40.0 \\
\hline Albumin (g/dL) & 4.2 & 4.0 & 4.1 & 4.2 & 3.7 & 3.9 \\
\hline
\end{tabular}

HD: hospital day, AST: aspartate phosphatase, ALT: alanine aminotransferase, ALP: alkaline phosphatase.

performed because the patient complained of peritonitis symptoms, and showed abdominal guarding, rigidity, and rebound tenderness, mainly in the right upper quadrant. Abdominal CT showed an increased amount of hemoperitoneum in the abdominal cavity, perihepatic fluid collection, and hemoperitoneal fluid (Fig. 3A). In addition, heterogeneous contrast filling in the gallbladder was observed without active bleeding (Fig. 3B). The patient underwent percutaneous gallbladder drainage using an 8-Fr pigtail catheter (Fig. 4A). Liver enzyme (AST and ALT) and bilirubin levels gradually decreased and normalized over 2 weeks (Table 1). After 27 days, the final abdominal CT before catheter removal showed a collapsed gallbladder over the catheter with resolution of perihepatic fluid collection (Fig. 4B). The patient's symptoms improved, and he was discharged without any complications.

\section{DISCUSSION}

Gallbladder injuries are mainly caused by penetrating injuries. Blunt abdominal trauma is associated with three categories of injury, ranging from contusion to lacerations and avulsions of the gallbladder, for which the most common mechanisms are traffic accidents (motor vehicle collisions, overturns, and rolling down) [1-4,7]. CT and laparoscopy are used to diagnose patients with traumatic gallbladder injuries. CT is most often used after trauma in stable or unstable patients to exclude associated visceral injuries. Simultaneous laceration of the liver and gallbladder injury are rare due to blunt trauma, and although this combination is fatal if the injury also affects the cystic artery.
Emergency laparotomy (laparoscopic approach) with cholecystectomy, drainage of hematomas with percutaneous gallbladder drainage using ultrasonography-guided catheters, and peritoneal lavage have been performed in many reported cases [8-10]. However, trans-arterial embolization (TAE) has been proposed and challenged as an alternative non-operative treatment option $[10,11]$. TAE is included as a step involving a quick and concise treatment process in patients with severe trauma to minimize surgical treatment. However, gallbladder necrosis can occur as a rare complication after hepatic trans-catheter angioembolization due to inadvertent embolization of the cystic artery $[6,10,11]$. This complication is typically caused by non-targeted embolization of the cystic artery during embolization of the right hepatic artery. In this case, active bleeding in the gallbladder lumen and persistent bile leakage in percutaneous drainage (PCD) were observed. If persistent bile leakage from the gallbladder to the peritoneal cavity occurred, we might have considered surgical options, such as exploratory surgery or laparotomy. If embolization was required, TAE might have been performed to prevent focal contrast extravasation on cystic arteriography. However, for the liver injury, only selective embolization of the right hepatic artery was performed, and the bleeding from the cystic artery was observed. This choice was made to avoid complications such as gallbladder necrosis, which is more likely to occur when embolization of the cystic artery is performed. In addition, gallbladder bleeding may be stopped due to normal hemostasis, the compression effect from the hematoma, and the effects of anti-fibrinolytic therapy. The patient underwent physical examination and hemody- 
namic monitoring in the intensive care unit to determine whether abdominal pain, which may lead to the suspicion of bile peritonitis, occurred. In conclusion, although angiography may show bleeding findings in cases of traumatic gallbladder injuries, close observation may be possible in the intensive care unit rather than immediate embolization. In addition, when cholecystitis symptoms are noted, non-operative management using PCD may be considered.

\section{REFERENCES}

1. Egawa N, Ueda J, Hiraki M, Ide T, Inoue S, Sakamoto Y, et al. Traumatic gallbladder rupture treated by laparoscopic cholecystectomy. Case Rep Gastroenterol 2016;10:212-7.

2. Hamilton C, Carmichael S, Bernard A. Laparoscopic cholecystectomy for traumatic gallbladder perforation. J Surg Case Rep 2012;2012:13.

3. Philipoff AC, Lumsdaine W, Weber DG. Traumatic gallbladder rupture: a patient with multiple risk factors. BMJ Case Rep 2016;2016:bcr2016216811.

4. Salzman S, Lutfi R, Fishman D, Doherty J, Merlotti G. Traumat- ic rupture of the gallbladder. J Trauma 2006;61:454-6.

5. Huang CC, Lo HC, Tzeng YM, Huang HH, Chen JD, Kao WF, et al. Percutaneous transhepatic gall bladder drainage: a better initial therapeutic choice for patients with gall bladder perforation in the emergency department. Emerg Med J 2007;24:836-40.

6. Latremouille R, Martinez-Quinones P, Lopez NS, White CQ. Gallbladder necrosis after right hepatic artery embolization due to traumatic hepatic injury. Am Surg 2018;84:e237-8.

7. Sharma O. Blunt gallbladder injuries: presentation of twenty-two cases with review of the literature. J Trauma 1995;39:57680.

8. Søndenaa K, Horn A, Nedrebø T. Diagnosis of blunt trauma to the gallbladder and bile ducts. Eur J Surg 2000;166:903-7.

9. Zerem E, Omerovic S, Kunosic S. Sonographically guided percutaneous treatment of liver abscesses in critically Ill patients. J Clin Ultrasound 2014;42:527-33.

10. Misselbeck TS, Teicher EJ, Cipolle MD, Pasquale MD, Shah KT, Dangleben DA, et al. Hepatic angioembolization in trauma patients: indications and complications. J Trauma 2009;67:769-73.

11. Green CS, Bulger EM, Kwan SW. Outcomes and complications of angioembolization for hepatic trauma: a systematic review of the literature. J Trauma Acute Care Surg 2016;80:529-37. 